\title{
Silencing by small RNAs is linked to endosome trafficking
}

\author{
Young Sik Lee ${ }^{1,2}$, Sigal Pressman ${ }^{1}$, Arlise P. Andress ${ }^{1}$, Kevin Kim ${ }^{1}$, Jamie L. White ${ }^{1}$, Justin \\ J. Cassidy ${ }^{1}$, Xin Li ${ }^{1,4}$, Kim Lubell ${ }^{1}$, Do Hwan Lim ${ }^{2}$, Ik Sang Cho ${ }^{2}$, Kenji Nakahara ${ }^{1,3}$, \\ Jonathan B. Preall ${ }^{1}$, Priya Bellare ${ }^{1,5}$, Erik J. Sontheimer ${ }^{1}$, and Richard W. Carthew ${ }^{1}$ \\ ${ }^{1}$ Department of Biochemistry, Molecular Biology and Cell Biology, Northwestern University, \\ Evanston, Illinois 60201 USA \\ ${ }^{2}$ College of Life Sciences and Biotechnology, Korea University, Seoul 136-713, South Korea
}

\section{Abstract}

\begin{abstract}
Small RNAs direct RNA induced silencing complexes (RISCs) to regulate the stability and translation of mRNAs1,2. RISCs associated with target mRNAs often accumulate in discrete cytoplasmic foci known as GW-bodies3. However, RISC proteins can associate with membrane compartments such as the Golgi and ER4. Here, we show that GW-bodies are associated with late endosomes or multivesicular bodies (MVBs). Blocking turnover of MVBs into lysosomes by loss of the tethering factor HPS45, enhances siRNA- and miRNA-mediated silencing in Drosophila and humans. It also triggers over-accumulation of GW-bodies. Blocking MVB formation by ESCRT6 depletion results in impaired miRNA silencing and loss of GW-bodies in cells. These results indicate that active RISC is physically and functionally coupled to MVBs. We further show that MVBs promote the competence of RISC to load small RNAs. We suggest that recycling of RISC is promoted by MVBs in order to more effectively engage with small RNA effectors and possibly target RNAs. It may provide a means to enhance the dynamics of RNA silencing in the cytoplasm.
\end{abstract}

\begin{abstract}
We carried out a mutagenesis screen in Drosophila to identify mutations that enhance siRNA-mediated gene silencing7. One complementation group contained five mutant alleles that displayed enhanced silencing of the white gene (Fig. 1a-c). The mutants did not display any other detectable phenotype. To confirm that the mutants generally enhanced silencing, we examined siRNA-mediated silencing of a different gene. Loss of $C s k$ in eye tissue results in apoptosis of eye cells8. When Csk dsRNA was expressed in the eye, there was a weak RNAi effect (Fig. 1d-e). However, eye size was greatly reduced in the mutants (Fig. 1f), indicating that $C s k(\mathrm{RNAi})$ was enhanced. We further tested whether the mutants enhanced miRNA-mediated silencing. We used a GFP reporter transgene linked to the $3^{\prime}$ UTR of the
\end{abstract}

\footnotetext{
Users may view, print, copy, and download text and data-mine the content in such documents, for the purposes of academic research, subject always to the full Conditions of use:http://www.nature.com/authors/editorial_policies/license.html\#terms

Correspondence should be addressed to Y.S.L. (ys-lee@korea.ac.kr) and R.W.C. (r-carthew@ northwestern.edu).

${ }^{3}$ Hokkaido University, N9, W9, Kita-ku, Sapporo, 060-8589 Japan

${ }^{4}$ Department of Biology, New York University, New York, NY, USA

${ }^{5}$ Department of Microbiology \& Immunology, Howard Hughes Medical Institute, University of California at San Francisco, San Francisco, CA, USA

Supplementary Information

Six supplementary figures (SFig. 1 to SFig. 6) are associated with this manuscript.
} 
$B r d$ gene, which contains a number of miRNA binding sites9. Expression of the GFP ::Brd reporter is repressed in a miRNA-dependent manner (Fig. 1g,h). We examined reporter expression in animals that contained clones of mutant cells amidst wildtype cells. Eyes showed patches of weaker-than-normal GFP fluorescence, indicating enhanced repression (Fig. 1i). Together, these results indicate that the mutants enhance both siRNA and miRNA silencing pathways in Drosophila. Although these RNAi pathways were elevated in the mutants, this was not simply due to an increase in abundance of known protein components of these pathways (SFig. 1).

We positionally cloned the mutated gene to be CG4966, which is orthologous to the Hermansky-Pudlak Syndrome 4 (HPS4) gene (Fig. 1j). Hermansky-Pudlak Syndrome (HPS) is a rare group of autosomal recessive diseases5. Its etiology has been related to defects in seven genes, all of which act in the endosome pathway that forms lysosome-related organelles. The yeast HPS4 orthologue Ccz1 together with the HPS1 orthologue Mon1 promotes tethering of late endosomes for fusion to the vacuole10. In mouse cells, a complex of HPS4 and HPS1 proteins is required for fusion of late endosomes with lysosomes and related organelles5,11,12. The presence of SNARE-like domains in HPS4 and HPS1 supports a tethering function in the fusion process 13. To determine if Drosophila HPS4 (dHPS4) protein behaves like its mammalian orthologue, we examined its membrane association and found that it is enriched around lysosomes and late endosomes (Fig. 2a-c). Moreover, it associates with the Drosophila HPS1 orthologue (dHPS1) when co-expressed in $\mathrm{S} 2$ cells (Fig. 2d-e).

We wondered if loss of HPS4 can also enhance RNAi in mammalian cells. HPS1 and HPS4 transcript levels were knocked down in cultured HeLa cells using siRNA treatment (SFig. 2a-c). We then tested whether knockdown affected RNAi in treated cells, as measured by silencing of a luciferase reporter. We observed a two-fold increase in luciferase silencing when HPS4 alone or HPS1/4 were knocked down, compared to control-treated cells (Fig. 1k and SFig. 2e,f). From these experiments, we conclude that HPS4 attenuates RNAi across the animal kingdom.

Does HPS4 affect RNAi because of its role in endosome trafficking? To explore this issue, we disrupted other steps in the endosome trafficking pathway. Normally, cargo from early endosomes arrives at the late endosome en route to the lysosome6. In this transition, regions of the endosomal limiting membrane invaginate into the interior and form lumenal vesicles, generating a late endosome known as the multivesicular body (MVB). The mature MVB packed with vesicles fuses with a lysosome to deliver its cargo. It is this turnover step where HPS4 acts. Upstream of this step, the formation of MVBs requires a number of ESCRT proteins to direct the process 14 . We eliminated ESCRT factors using mutants, and observed the effects on RNA-mediated silencing. The ESCRT genes hrs and vps 25 are required for maturation of early endosomes in Drosophila15-17. We observed impaired miRNAmediated silencing of the GFP ::Brd reporter in hrs and vps25 mutant cells (Fig. 3a-c). A different reporter for miRNA-mediated silencing was also de-repressed in vps 25 mutant clones (Fig. 3d-e). Altogether, these data indicate that blocking MVB formation (with ESCRT mutants) inhibits silencing while blocking MVB turnover (with HPS4 mutants) 
stimulates silencing. These results implicate MVBs as important compartments for RNA silencing.

The miRNA pathway involves the loading of a miRNA into RISC (miRISC), and the active association of miRISC with target mRNAs. We visualized the distribution of all miRISC complexes (active, loaded, and inactive) in Drosophila cells using an antibody recognizing the Agol protein, a key subunit of miRISC. Ago1 was detected in the cytoplasm of cells, and was enriched in the perinuclear region (Fig. 3f), where lysosomes tend to localize4. In dHPS4 mutant cells, Ago1 was not enriched in the perinuclear region but was more dispersed throughout the cytoplasm (Fig. 3g). This result suggests that Ago1 localization correlates with trafficking endosomes. As further evidence, cells mutant for $v p s 25$ and the ESCRT regulator myopic17 showed Ago1 concentrated around large cytoplasmic vesicles (Fig. 3h-i). These mutations generate cells with enlarged early endosomes because they block sorting of early endosomes into MVBs16,17. Hence, the distribution of miRISC appears to correlate with the status of MVBs; blocking MVB formation concentrates miRISC in early endosomes while blocking MVB turnover disperses miRISC from lysosomes. Even though Ago1 is dispersed in dHPS4 cells, it is still associated with membranes in the cytoplasm (SFig. 3a). We separated cytoplasmic membrane from cytosol by OptiPrep density gradient centrifugation, and found a substantial fraction of Ago1 protein associated with membrane. There was little change in its association with membrane in dHPS4 mutant preparations. A similar result was observed with Ago2 protein (SFig. 3a).

One or more features of miRISC could possibly be regulated by MVBs. We examined miRISCs actively associated with target mRNAs, which are concentrated in cytoplasmic foci called GW-bodies3. We visualized the distribution of GW-bodies in Drosophila S2 cells using a YFP-GW182 fusion protein19. YFP-GW182 bodies were closely juxtaposed to the cytosolic face of MVBs and/or lysosomes, as marked by the Lamp1 protein14 (Fig. 3j). Quantitative analysis indicated that $60 \%$ of GW-bodies were associated with Lamp1 vesicles in this manner (SFig. 3b). We calculated that the frequency of association that would occur by chance was about $4 \%$, and the observed frequency of association was highly unlikely to have occurred by chance alone ( $p<0.001$ in two-tailed $\chi 2$ test). Another marker of active silencing complexes, GFP-Me31b 20, was also associated with Lamp1-positive vesicles (Fig. 3k). Thus, GW-bodies associate with MVBs or lysosomes.

To determine if these bodies are regulated by MVBs, we looked in HeLa cells that were depleted for ESCRT factors or HPS4 (SFig. 2d), using the mammalian miRISC subunit Ago2 as a marker for GW-bodies3. Depletion of Hrs and the ESCRT-II factor Tsg101 resulted in fewer bodies (Fig. 31). In contrast, depletion of HPS1/4 resulted in increased numbers of these bodies. All differences were statistically significant $\left(p<10^{-8}\right.$ by Kolmogorov-Smirnov test). These results implicate MVBs as critical for formation and/or stability of GW-bodies. Together with the localization data, they suggest that MVBs are important sites of miRISC association with target mRNAs.

It is also possible that MVBs regulate other aspects of miRISC such as its loading with miRNAs. We examined the level of miRNA loading into miRISC by immunoprecipitating Drosophila Ago1 and measuring the levels of different miRNAs associated with Ago1. 
When we did so, we readily detected miRNAs from wildtype cells (Fig. 4a). The levels of these miRNAs were greater when Ago1 was immunopurified from dHPS4 mutant cells, arguing that miRISC loading is enhanced when MVB turnover is impaired. This enhanced loading was not simply due to greater abundance of small RNAs in dHPS4 mutants (SFig. 4 and data not shown). We also tested for the competence of miRISC to load miRNAs de novo. This was assayed by adding an exogenous miRNA to extracts derived from Drosophila, and measuring the level of loaded miRISC by its cleavage activity against a target mRNA. The activity of miRISC programmed by the exogenous miRNA was enhanced several-fold in extracts from $d H P S 4$ mutants (Fig. 4b). This result suggests that the competence to load miRNAs into miRISC is enhanced when MVB turnover is impaired.

Silencing mediated by siRNAs is also enhanced in dHPS4 mutants (Fig. 1a-f). Is this effect mechanistically related to the effects of $d H P S 4$ on miRISC? To investigate, we added an exogenous siRNA to Drosophila extract and measured siRNA-guided RISC (siRISC) cleavage activity against a target mRNA (Fig. 4b). Activity was enhanced three-fold in dHPS4 mutant extract. To confirm that enhanced activity was due to greater competence of siRISC to load siRNA, we measured loading of labelled exogenous siRNA. When added to cytoplasmic extract, exogenous siRNA rapidly associates with Dicer-2 and R2D2 proteins to form the RDI complex, which matures to the RISC Loading Complex (RLC)18. Next, the siRNA duplex is loaded onto siRISC, initially forming a pre-RISC complex 21 . The siRNA is unwound within siRISC to form a complex that is fully capable of cleaving target transcripts (holo-RISC)18. In $d H P S 4$ mutant extract, pre-RISC and holo-RISC complexes were loaded at two- to three-fold higher levels with exogenous siRNAs (Fig. 4c,d). Kinetic analysis indicated that $d H P S 4$ mutant extract loaded siRISC more rapidly (Fig. 4e). The initial rates of RDI and RLC formation were nevertheless normal. To confirm that the step of loading siRISC from RLC is affected, we pre-loaded siRNA onto purified RDI. This was chased into siRISC with addition of extract. Under these pulse-chase conditions, we observed a greater level of siRISC loading upon addition of mutant extract (Fig. 4f). In summary, the competence of siRISC loading is enhanced when MVB turnover is impaired.

To confirm that siRISC loading is positively regulated by MVBs, we inhibited MVB formation. Depletion of both Tsg101 and Hrs weakly impaired siRNA-mediated silencing, though depleting either one alone had no effect (SFig. 5). Since different sorting-related phenomena are differentially regulated by various ESCRTs6,14, we considered that siRISC might rely upon ESCRTs that are different from those that affect miRISC. Therefore, we inhibited MVB formation in an alternative way. Maturation of early endosomes into MVBs critically depends upon recognition of ubiquitinated proteins in early endosomes by ESCRT proteins14. Hence, we predicted that siRISC would depend upon protein ubiquitination. From our mutagenesis screen, we had isolated a missense mutation in the Drosophila orthologue of FBX011, a subunit of a SCF-like ubiquitin ligase22,23. The mutant displayed impaired silencing mediated by both siRNAs and miRNAs (Fig. 5a-f). Yet, $d F B X 011$ mutants contained normal levels of small RNAs, arguing for a silencing defect downstream of RNA processing (Fig. $5 \mathrm{~g}-\mathrm{h}$ ). The effects of this mutant and another mutant allele of dFBX011 on siRISC activity were examined in vitro. In mutant extracts, siRISC loading 
with an exogenous siRNA was much less active than normal (Fig. 5i). Thus, siRISC activity relies upon the presence of a ubiquitin ligase.

Ubiquitin-binding proteins interact with a hydrophobic patch on the surface of ubiquitin, and an I44A substitution in ubiquitin blocks most of these interactions. This can exert a dominant-negative effect on ubiquitin-dependent processes in vitro24. When an excess of purified I44A mutant ubiquitin was added to Drosophila extract, loading of siRISC with exogenous labelled siRNA was dramatically inhibited (Fig. 5j). No effects were detected on RDI and RLC. The effect on siRISC loading was lost with an I44A derivative ubiquitin that could not enter the conjugation pathway due to a four-aminoacid C-terminal deletion24. These data together suggest that protein ubiquitination stimulates RISC loading, consistent with the hypothesis that RISC loading is positively regulated by MVBs. A caveat to this interpretation is that ubiquitin affects numerous processes, chief among them protein degradation. However, proteasome inhibitors had no effect on siRISC loading, suggesting that ubiquitin does not regulate siRISC through protein degradation (SFig. 6a). Although effects through additional ubiquitin pathways cannot be excluded, the data is wholly consistent with our other work implicating a link between MVBs and RISC loading.

We propose that MVBs promote RNA-mediated silencing in two non-mutually-exclusive ways. They recruit active miRISC complexes associated with target mRNAs into GWbodies that are physically associated with MVBs. If MVBs do not form, there is a buildup of miRISC associated with early endosomes accompanied by a dropoff in active miRISC complexes associated with GW-bodies. Therefore, the very engagement of miRISC with target mRNAs might be coupled to MVB formation (SFig. 6b). A second way that MVBs regulate silencing is by increasing miRISC competence to load miRNAs, perhaps by promoting turnover of miRISC (SFig. 6b). This model is consistent with another study implicating MVBs as sites of miRISC turnover due to GW182 sorting25. We observe a similar mechanistic link between MVBs and siRISC loading as well. However, we do not know if target mRNAs associated with siRISC also localize to MVBs.

Why is RNAi linked to endosome trafficking? RNA viruses use the endocytic pathway for entry, replication and release26, and siRNAs are used for defense against virus infection 27. Localization of RISC machinery to sites inhabited by viruses may enhance the antiviral response. Another explanation is that MVBs might recycle RISC complexes active at peripheral sites of cells such as neurons28. MVBs transport cargo from synapses to cell bodies29, and so they may enable RISC to shuttle target mRNAs away from the periphery and allow RISC to re-engage with newly synthesized small RNAs. Finally, it could provide a means for small RNAs to be secreted from cells via the exosome pathway, either for possible immune or regulatory purposes30. Indeed, small RNAs have been detected within exosomes generated from MVBs and secreted from cells25,31.

\section{Methods}

\section{Drosophila genetics}

Mutant alleles of dicer- 1 and dicer-2 have been described7. The dsRNA-generating transgene targeting white, referred to as GMR-wIR, has also been described7. The dsRNA- 
generating transgene targeting $C s k$, referred to as $C s k(\mathrm{RNAi})$, was kindly provided by $\mathrm{R}$. Cagan8. The $h r s^{D 28}$, vps $25^{A 3}$, and myopic ${ }^{T 612}$ mutations have been described15-17.

The eyFLP/FRT system 32 was used to make clones of mutant clones of cells by means of eye-specific mitotic recombination. These generated homozygous mutant clones for $d H P S 4$, dFBX011, dicer-1, vps25, myopic, and hrs. Clones were marked with $U b>G F P$ or $a r m>l a c Z$ cell autonomous markers, when possible. Clones were assayed for siRNA-mediated silencing activity against the white gene by GMR-wIR. Clones were also assayed for miRNA-mediated silencing activity with transgenic reporters. One reporter is arm>lacZ::E(spl)m8 that was previously described9. The other reporter is a GFP reporter gene. We constructed it by placing the GFP-coding sequence followed by the $3^{\prime}$ UTR of the Bearded gene into the pGMR transformation vector. The GMR promoter drives expression primarily in the differentiating eye. Transgenic flies in a $w^{1118}$ mutant background were generated by standard procedures. No mini-white marker was used for transgenesis since pigmentation interferes with GFP fluorescence.

Complementation analysis and genetic mapping of mutants were performed as described7. The molecular lesions in the coding regions of either $d H P S 4$ or $d F B X 011$ genomic DNA were determined by sequencing PCR products amplified from $d H P S 4$ and $d F B X 011$ mutant animals. The $d F B X 011^{37}$ mutation was generated by imprecise excision of the $\mathrm{P}$ element in

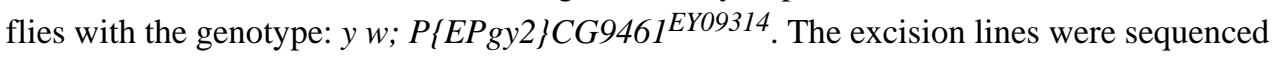
to determine the nature of the mutation.

\section{Mutagenesis Screen}

The mutagenesis screen that identified alleles of $d H P S 4$ and $d F B X 011$ was performed as previously described7. We screened for EMS-induced mutations that resulted in reduced or enhanced RNAi activity in Drosophila. Clones of homozygous mutant cells were generated in the developing eyes of heterozygous F1 animals. Since the wild-type chromosome was marked with an eye-specific cell-death gene, only homozygous mutant eye cells were present in adults. This approach enabled us to recover mutations that otherwise were homozygous lethal or sterile to the animal. The mosaic F1 animals also carried a transgene $(G M R-w I R)$ that silenced expression of the endogenous white gene7. GMR-wIR lightens wildtype eye pigmentation from bright red to light orange. Since one copy of GMR-wIR does not completely silence white, we were able to compare the eye pigmentation of GMR$w I R$ in wild-type animals (pale orange) with those of mutants, looking for enhanced (white) and suppressed (red) phenotypes. Our screen of the $3 \mathrm{R}$ and $2 \mathrm{R}$ autosome arms isolated the mutations in $d F B X 011$ and $d H P S 4$, respectively.

\section{Antibodies}

Polyclonal anti-dHPS4 antiserum was generated by immunization of guinea pigs with recombinant GST-fusion protein (Harlan Bioscience). The fusion protein contained aminoterminal GST in frame with the carboxyl-terminal 336 amino acids of dHPS4. Monoclonal anti-dFMR antibody was obtained from the Developmental Studies Hybridoma Bank. Other antibodies used were: anti-Dcr-2 (a gift from Q. Liu), anti-dFMR, anti-TSN, anti-VIG (gifts from G. Hannon), anti-Ago2 (a gift from M. Siomi), anti-Dcr-1 (Abcam), anti-Ago1 (Abcam 
and a gift from M. Siomi), and anti-s-tubulin (Developmental Studies Hybridoma Bank). Anti-dGM130 antibody was a gift from M. Lowe. Anti- $\beta$-Gal was a gift from G. Beitel. Xgal staining and immunohistochemistry of Drosophila tissues was performed as described7. Specificity of Ago1 immunoreactivity was verified by staining eye discs bearing clones of Agol mutant cells. These clones lacked staining with the antibody (data not shown).

\section{Confocal Microscopy}

A Zeiss 510 Meta confocal microscope system was used to acquire images. Optical sections ranging from 0.4 to $1.0 \mu \mathrm{m}$ were collected as a Z-series through samples. Images of S2 cells utilized a 100X objective, whilst images of Drosophila tissue used 20X and 100X objectives. Images were scanned at 1024 or 2048 pixel resolution. Gain levels and total number of images within each stack were kept constant if possible.

\section{S2 cell culture and immunofluorescence}

To express epitope-tagged dHPS4 and dHPS1 proteins in S2 cells, the coding regions were triple-HA- or triple-FLAG-tagged at the amino termini and cloned into the expression vector pMK33 that has an inducible metallothionein promoter. Plasmids were also constructed constitutively expressing GFP-Me31b and YFP-GW182. Lamp1-RFP plasmid was a gift of G. Goshima (Nagoya University).

Stable cell lines containing MT>HA-dHPS4 were selected by hygromycin. Expression from the metallothionein promoter was induced by the addition of $\mathrm{CuSO}_{4}$ to a final concentration of $0.7 \mathrm{mM}$. A stable line with constitutive Lamp1-GFP expression was a gift of V. Gelfand (Northwestern University).

$\mathrm{S} 2$ cells transfected with expression plasmids were harvested $24 \mathrm{~h}$ after induction of expression. At least 2 hours prior to fixation, cells were transferred onto poly-L-lysine coverslips. Cells were fixed with $2 \%$ paraformaldehyde in PBS and washed with $0.1 \%$ Saponin-PBS. Cells were incubated with 1:50 dilution of rat anti-HA antibody and 1:200 Alexa-546 anti-rat antibody.

\section{S2 Cell Imaging Analysis}

High resolution images $(2048 \times 2048)$ of 0.4 um thick optical sections were captured. Image analysis was performed in Adobe Photoshop CS3 at 300 dpi. The Magic Wand Tool was used to highlight each cell's cytoplasm, the YFP-GW182, and Lamp1-RFP bodies. A 1-pixel stroke was used to mark their boundaries. YFP-GW182 bodies were classified as being associated with Lamp1-RFP bodies if their boundaries were within 3 pixels of each other.

There is a probability that any single YFP-GW182 body is situated within 3 pixels from a Lamp1-RFP body by chance alone. To determine this probability, we calculated the total number of positions that an average YFP-GW182 body would occupy and be classified as Lamp1-RFP-associated. This number $N p$ was determined by tracing the path length surrounding each Lamp1-RFP body. We then created a shell surrounding the path, with the shell width being the average diameter of a YFP-GW182 body. We traced a 3 pixel path on 
the outer side of the shell, and measured this path length in pixel units. Each pixel in this outer path represents one possible position in which a YFP-GW182 body is associated with the Lamp1-RFP body. We summed the outer path lengths for all Lamp1-RFP bodies in a cell, and corrected for situations where neighboring Lamp1-RFP bodies created overlapping shells or shells were outside of the cell. This corrected value in pixels was used to define the number $N p$.

We also calculated the total number of positions that an average GW-182 body could occupy in the cell. To do this, we calculated the total number of pixels $N t$ occupying the cytosol (non-organelle cytoplasm). Nuclear pixels were subtracted from the total cell pixels to give cytoplasm pixels. Since a typical animal cell's cytosol accounts for $50 \%$ of the cytoplasm33, the number of cytoplasm pixels was reduced by $50 \%$ to derive $N t$.

The ratio of $N p / N t$ represents the fraction of positions that a YFP-GW182 body residing in the cytosol would be considered as being associated with a Lamp1-RFP body if its localization was random. The mean ratio of $N p / N t$ was found to be 0.044 (range 0.023 to 0.070) for 105 Lamp1-RFP bodies. In contrast, the observed fraction of YFP-GW182 bodies classified as being associated with Lamp1-RFP bodies was found to be 0.599 (range 0.300 to 0.818 ) for 67 YFP-GW182 bodies. To determine the statistical significance of the difference between observed and chance, we performed a two-tailed binomial $\chi 2$ test. The significance is $p<0.001$.

\section{RNA analysis}

RT-qPCR was performed on cellular RNA using a Bio-Rad iCycler. HPS1: GGGCAGTCAGAGAATGAGGA and GATGACCGGGGCTAGGAG; HPS4: AAAGTTGATGGAGATTACCTTTGG and CTGATCCAGAAACCGCTTG; GAPDH: GAAGGTGAAGGTCGGAGT and GAAGATGGTGATGGGATTTC. Northern blot analyses for $G M R$-wIR hairpin RNA, its derived siRNAs, and miR-2 were performed with total RNA isolated from heads as described7.

\section{Drosophila extract preparation}

To prepare Drosophila head extracts, adult heads were collected using sieves by standard procedures. Heads were homogenized in $0.4 \mu \mathrm{l}$ lysis buffer (30 mM HEPES-KOH pH 7.4, $100 \mathrm{mM}$ potassium acetate, $2 \mathrm{mM}$ magnesium acetate, $5 \mathrm{mM}$ DTT and $1 \mathrm{mg} / \mathrm{ml}$ Pefabloc SC) per head. The homogenate was clarified by centrifugation at $14,000 \times \mathrm{g}$ for $15 \mathrm{~min}$. Embryo extracts were prepared as described previously 18.

\section{Ago1 immunoprecipitation and measurement of associated miRNAs}

Head extracts were prepared in $30 \mathrm{mM}$ HEPES-KOH, $\mathrm{pH} 7.4,100 \mathrm{mM}$ potassium acetate, 2 $\mathrm{mM}$ magnesium acetate, $5 \mathrm{mM}$ DTT, and $0.1 \%$ Nonidet P-40 supplemented with protease inhibitors (Roche). To immunoprecipitate Ago1, head extracts were incubated with monoclonal anti-Ago1 (1B8) (a gift from M. Siomi) coupled to Dynabeads at $4^{\circ} \mathrm{C}$ for $2 \mathrm{~h}$ with gentle agitation. As a control, extracts were immunoprecipitated with anti-GFP in parallel. Bound RNA was isolated from the immunoprecipitate by deproteinizing, phenol/ chloroform extraction, and ethanol precipitation. Levels of miR-8, miR-276a and miR-277 
were quantitatively analyzed by splinted ligation using an miRtect-IT miRNA labeling and detection kit (USB) according to the manufacturer's instructions. MiRNA-specific bridge oligonucleotides were as follows;

miR-8, 5'-GAATGTCATAAGCGGACATCTTTACCTGACAGTATTA-3'

miR-276a, 5'-GAATGTCATAAGCGAGAGCACGGTATGAAGTTCCTA-3'

miR-277, 5'-GAATGTCATAAGCGTGTCGTACCAGATAGTGCATTTA-3'

Reaction products were resolved on a $12 \%$ polyacrylamide $7 \mathrm{M}$ urea gel, visualized on a BAS-2500 Phosphorimager (Fujifilm), and quantified using MultiGauge software. RNA from anti-GFP immunoprecipitates did not generate any detectable miRNA splint-ligation products, demonstrating the specificity of the co-IP with anti-Ago1. Total RNA was also used to examine the expression of the miRNAs in the extracts, and they were found to be unchanged between wildtype and dHPS4 mutant. For each sample, an aliquot of Ago1 immunoprecipitate was subjected to Western blot analysis, and the levels of Ago1 were quantified with a LAS-3000 chemiluminescence imaging system (Fujifilm). Levels of RNA were normalized to the level of Ago1 protein that was immunoprecipitated for each sample.

\section{In vitro RNAi assays}

Target RNA cleavage with either head or embryo extracts were performed as previously described18. The sequences for bantam miRNA used to guide RISC activity are as follows:

bantam sense: 5'-CGGUUUUCGAUUUGGUUUGACU-3'

bantam antisense: 5'-UGAGAUCAUUUUGAAAGCUGAUU-3'.

Experiments to assay RISC loading were carried out as described previously18,21. To detect pre-RISC complex in head extracts, we used an siRNA with a single base mismatch that is designated 5'-PUR siRNA21. This siRNA allows pre-RISC detection in head extracts though it does not have the same effect in embryo extracts (unpublished data). Otherwise, $P p$-Luc siRNA was used in experiments. For pulse-chase experiments, R2D2/Dicer-2 heterodimer was partially purified from wild-type embryos, and reactions were carried out as previously described18. Chase reactions were performed with head extracts.

\section{Western blot and immunoprecipitations}

To compare the levels of various RNAi proteins in wildtype, $d H P S 4^{W 515 X}$ and $d H P S 4^{E 133 X}$ homozygotes, head extracts were prepared from adults of each genotype. Blots were subsequently incubated with HRP-conjugated goat secondary antibody (Bio-Rad), and processed for chemiluminescence. Chemiluminescense was detected using the LAS-3000 imaging system (Fujifilm) and quantified using MultiGauge software.

For co-IP assays, cells were lysed in $10 \mathrm{mM}$ sodium phosphate $\mathrm{pH} 7.2,150 \mathrm{mM} \mathrm{NaCl}, 1 \%$ Nonidet P-40, $2 \mathrm{mM}$ EDTA, $50 \mathrm{mM} \mathrm{NaF}, 200 \mathrm{mM} \mathrm{Na}_{3} \mathrm{VO}_{4}$ and protease inhibitors (Roche), and centrifuged at $14,000 \times \mathrm{g}$ for $15 \mathrm{~min}$. Clarified extract was incubated with 
mouse anti-HA antibody and Protein A agarose beads. After washing in lysis buffer, the beads were boiled and eluted proteins were used for Western blot analysis.

\section{OptiPrep density gradient centrifugation}

For linear density gradients, 200-300 mg heads were homogenized in $400 \mu \mathrm{l}$ lysis buffer containing $0.25 \mathrm{M}$ sucrose. Extracts were clarified three times by centrifugation at $1000 \times \mathrm{g}$ for $5 \mathrm{~min}$. The supernatant was diluted to a final volume of $3.4 \mathrm{ml}$ with lysis buffer containing sucrose and mixed with $5.1 \mathrm{ml}$ of working solution (5 volumes of OptiPrep34 with 1 volume of $6 \mathrm{X}$ lysis buffer). Samples were centrifuged for $1 \mathrm{~h}$ at $350,000 \times \mathrm{g}$ at $4{ }^{\circ} \mathrm{C}$, and $12 \times 600-\mu l$ fractions were collected. Aliquots of each fraction were analyzed by Western blotting.

\section{Depletion experiments in HeLa cells}

HeLa cells were maintained in DMEM $+10 \%$ fetal bovine serum. We also used a HeLa cell line (a gift from L. Aleman and P. Sharp) containing a stably integrated Renilla-luciferase (Ren-luc) gene with two modified CXCR4 binding sites in the $3^{\prime}$ UTR35. The targeting sequence is $5^{\prime}$ GUUUUCAUCUAGCUAACA $3^{\prime}$. The cell line also expresses an EGFPAgo2 gene.

The siRNAs against Tsg101 and Hrs were purchased from Dharmacon as ON-TARGETplus SMARTpools. Other siRNAs were purchased from IDT. The passenger strand sequences from $5^{\prime}$ to $3^{\prime}$ are listed as follows. Guide strands are perfectly complementary and are not listed. Most $3^{\prime}$ overhang sequences were GU or AU.

CXCR4 modified: GUUUUCACUCUAGCUAACACA

Control siRNA: GGUUGCUGGAGCUCUAAUU

$\begin{array}{lll}\text { HPS1: } & & \\ \text { Number } & \text { Start position } & \text { Sequence } \\ 1 & 443 & \text { AGAAGCUCUCGGACACCCCA } \\ 2 & 464 & \text { CCUGCUUCUCCACGGAAAA } \\ 3 & 1001 & \text { GCACAGCAGAGGACGACAU } \\ 4 & 489 & \text { CUUCCUGUAUGUCCUUCAC } \\ 5 & 310 & \text { GAUCAGGAGUUUGAAGAGA } \\ 6 & 351 & \text { GUCAGAGAAUGAGGAAGAA } \\ 7 & 242 & \text { CUGUGCUUGCCAAGAUGAA } \\ 8 & 413 & \text { CGGUCAUCAUCUCCUCCAU } \\ 9 & 284 & \text { GCGCAGAGGUCCUCUUUCU } \\ 10 & 962 & \text { CCCUCAUCCUCCUGGUUCA } \\ \text { HPS4: } & & \\ \text { Number } & \text { Start position } & \text { Sequence } \\ 1 & 1017 & \text { CCAGUGAUCUGCAUAAGAU } \\ 2 & 629 & \text { UAUGAUGGUUCCAAGGUAA } \\ 3 & 803 & \text { AAACUGAAGUUUGCCAUAA } \\ 4 & 652 & \text { AGAAGGCGAUCCAACAAGA }\end{array}$




$\begin{array}{cll}5 & 882 & \text { GCAAGCGGUUUCUGGAUCA } \\ 6 & 1718 & \text { GAACUCGACUUGUCUGAAA } \\ 7 & 1696 & \text { GGAACUAGUCUUUCUCCAA } \\ 8 & 1204 & \text { GGUCCUGCUUCCCGAACA } \\ 9 & 1510 & \text { CGCCACUGGCCAUGUGGAA } \\ 10 & 1348 & \text { UGUCCAGAUUAUCCCUGUU } \\ 11 & 746 & \text { UACAUUUCAAAAUGUGCCG }\end{array}$

Cells at $60-80 \%$ confluence were transfected with SilentFect (Bio-Rad). Unless otherwise stated, siRNAs were added to a final concentration of $10 \mathrm{nM}$. Cells were incubated for $8 \mathrm{~h}$, and then incubated with a second round of siRNA/SilentFect medium for 16h. Medium was replaced, and cells were allowed to recover for $24 \mathrm{~h}$ or more.

\section{Luciferase Assays}

Two approaches for siRNA-mediated silencing of luciferase reporters were performed. The first involved the HeLa cell line that stably expressed Ren-luciferase. This line was transfected with various combinations of control, CXCR4, Tsg101, Hrs, HPS1-242, and HPS4-1348 siRNAs. After 24h transfection and 24h post-transfection, cells were harvested and Ren-luciferase activities were assayed. Constant protein mass was added to reactions to normalize for extract variation.

The second approach involved a HeLa cell line transfected in two stages. In the first stage, dishes of cells were transfected with control or HPS4-882 siRNAs. Cells were split 1:2 in fresh medium and allowed to recover $48 \mathrm{~h}$. They were then transfected by Lipofectamine (Invitrogen) with plasmid CMV expression vectors for $F f$-luciferase, Ren-luciferase, and a shRNA directed against $F f$-luciferase sequences (all gifts of X. Wang). After 24h, cells were assayed for luciferase activities by the dual luciferase method. $F f$-luciferase activity was normalized to Ren-luciferase activity.

\section{Quantitation of EGFP-Ago2 cytoplasmic foci}

Cells were seeded onto glass slides and grown to $60-80 \%$ confluence. They were transfected with various combinations of control, Tsg101, Hrs, HPS1-242, and HPS4-1348 siRNAs. After $24 \mathrm{~h}$ transfection and $24 \mathrm{~h}$ post-transfection, cells fixed in $4 \%$ paraformaldehyde for 15 minutes. GFP fluorescent foci were visualized under $63 \mathrm{X}$ power with a Zeiss Axiophot. Although the majority of fluorescence is cytoplasmically diffuse, there are easily discernable foci in each cell36. These were scored blindly for all samples. A total of 400 cells were scored for each treatment.

\section{Supplementary Material}

Refer to Web version on PubMed Central for supplementary material.

\section{Acknowledgments}

We thank R. Cagan, H. Folsch, P. Sharp, G. J. Hannon, Q. Liu, X. Wang, C. Horvath, G. Goshima, V. Helfand, M. Lowe, I. Hariharan, D. Bilder, J. Treisman, M. Siomi, and J. Pham for reagents. We thank H. Folsch, I. Fields, A. Komuro, and D. Harris for help with some of the experiments. We thank H. Jiang for help with statistics. We also 
thank the Bloomington Stock Center and the Developmental Studies Hybridoma Bank for fly strains and antibodies. Y.S.L. was supported by a FRAXA postdoctoral Fellowship, J.J.C. was supported by the CMBD Training Grant, and J.B.P. was supported by a Presidential Fellowship. This work was also supported by grants from the National Institutes of Health to E.J.S. (GM072830) and R.W.C. (GM77581, GM68743), and from a grant from the Korean BioGreen 21 Program to Y.S.L. (20070301034036).

\section{References}

1. Zamore PD, Haley B. Ribo-gnome: the big world of small RNAs. Science. 2005; 309:1519-24. [PubMed: 16141061]

2. Carthew RW, Sontheimer EJ. Origins and mechanisms of miRNAs and siRNAs. Cell. 2009; 136:642-55. [PubMed: 19239886]

3. Filipowicz W, Bhattacharyya SN, Sonenberg N. Mechanisms of post-transcriptional regulation by microRNAs: are the answers in sight? Nat Rev Genet. 2008; 9:102-14. [PubMed: 18197166]

4. Cikaluk DE, et al. GERp95, a membrane-associated protein that belongs to a family of proteins involved in stem cell differentiation. Mol Biol Cell. 1999; 10:3357-72. [PubMed: 10512872]

5. Li W, et al. Murine Hermansky-Pudlak syndrome genes: regulators of lysosome-related organelles. Bioessays. 2004; 26:616-28. [PubMed: 15170859]

6. Piper RC, Katzmann DJ. Biogenesis and function of multivesicular bodies. Annu Rev Cell Dev Biol. 2007; 23:519-47. [PubMed: 17506697]

7. Lee YS, et al. Distinct roles for Drosophila Dicer-1 and Dicer-2 in the siRNA/miRNA silencing pathways. Cell. 2004; 117:69-81. [PubMed: 15066283]

8. Vidal M, Larson DE, Cagan RL. Csk-deficient boundary cells are eliminated from normal Drosophila epithelia by exclusion, migration, and apoptosis. Dev Cell. 2006; 10:33-44. [PubMed: 16399076]

9. Lai EC, Tam B, Rubin GM. Pervasive regulation of Drosophila Notch target genes by GY-box-, Brd-box-, and K-box-class microRNAs. Genes Dev. 2005; 19:1067-80. [PubMed: 15833912]

10. Wang CW, Stromhaug PE, Kauffman EJ, Weisman LS, Klionsky DJ. Yeast homotypic vacuole fusion requires the Ccz1-Mon1 complex during the tethering/docking stage. J Cell Biol. 2003; 163:973-85. [PubMed: 14662743]

11. Falcon-Perez JM, Nazarian R, Sabatti C, Dell'Angelica EC. Distribution and dynamics of Lamp1containing endocytic organelles in fibroblasts deficient in BLOC-3. J Cell Sci. 2005; 118:5243-55. [PubMed: 16249233]

12. Nguyen T, Wei ML. Hermansky-Pudlak HPS1/pale ear gene regulates epidermal and dermal melanocyte development. J Invest Dermatol. 2007; 127:421-8. [PubMed: 17068483]

13. Kinch LN, Grishin NV. Longin-like folds identified in CHiPS and DUF254 proteins: vesicle trafficking complexes conserved in eukaryotic evolution. Protein Sci. 2006; 15:2669-74. [PubMed: 17075139]

14. Williams RL, Urbe S. The emerging shape of the ESCRT machinery. Nat Rev Mol Cell Biol. 2007; 8:355-68. [PubMed: 17450176]

15. Lloyd TE, et al. Hrs regulates endosome membrane invagination and tyrosine kinase receptor signaling in Drosophila. Cell. 2002; 108:261-9. [PubMed: 11832215]

16. Vaccari T, Bilder D. The Drosophila tumor suppressor vps 25 prevents nonautonomous overproliferation by regulating notch trafficking. Dev Cell. 2005; 9:687-98. [PubMed: 16256743]

17. Miura GI, Roignant JY, Wassef M, Treisman JE. Myopic acts in the endocytic pathway to enhance signaling by the Drosophila EGF receptor. Development. 2008; 135:1913-22. [PubMed: 18434417]

18. Pham JW, Pellino JL, Lee YS, Carthew RW, Sontheimer EJ. A Dicer-2-dependent 80s complex cleaves targeted mRNAs during RNAi in Drosophila. Cell. 2004; 117:83-94. [PubMed: 15066284]

19. Behm-Ansmant I, et al. mRNA degradation by miRNAs and GW182 requires both CCR4:NOT deadenylase and DCP1:DCP2 decapping complexes. Genes Dev. 2006; 20:1885-98. [PubMed: 16815998]

20. Lin MD, et al. Drosophila processing bodies in oogenesis. Dev Biol. 2008 
21. Kim K, Lee YS, Carthew RW. Conversion of pre-RISC to holo-RISC by Ago2 during assembly of RNAi complexes. RNA. 2007; 13:22-9. [PubMed: 17123955]

22. Fielenbach N, et al. DRE-1: an evolutionarily conserved $\mathrm{F}$ box protein that regulates $\mathrm{C}$. elegans developmental age. Dev Cell. 2007; 12:443-55. [PubMed: 17336909]

23. Cardozo T, Pagano M. The SCF ubiquitin ligase: insights into a molecular machine. Nat Rev Mol Cell Biol. 2004; 5:739-51. [PubMed: 15340381]

24. Bellare P, et al. A role for ubiquitin in the spliceosome assembly pathway. Nat Struct Mol Biol. 2008; 15:444-51. [PubMed: 18425143]

25. Gibbings DJ, Ciaudo C, Voinnet O. Sorting of GW182 into multivesicular bodies controls microRNA activity. Nature Cell Biol. 2009 (in press).

26. Kujala P, et al. Biogenesis of the Semliki Forest virus RNA replication complex. J Virol. 2001; 75:3873-84. [PubMed: 11264376]

27. Ding SW, Voinnet O. Antiviral immunity directed by small RNAs. Cell. 2007; 130:413-26. [PubMed: 17693253]

28. Lugli G, Larson J, Martone ME, Jones Y, Smalheiser NR. Dicer and eIF2c are enriched at postsynaptic densities in adult mouse brain and are modified by neuronal activity in a calpaindependent manner. J Neurochem. 2005; 94:896-905. [PubMed: 16092937]

29. Parton RG, Simons K, Dotti CG. Axonal and dendritic endocytic pathways in cultured neurons. J Cell Biol. 1992; 119:123-37. [PubMed: 1527164]

30. Stoorvogel W, Kleijmeer MJ, Geuze HJ, Raposo G. The biogenesis and functions of exosomes. Traffic. 2002; 3:321-30. [PubMed: 11967126]

31. Valadi $\mathrm{H}$, et al. Exosome-mediated transfer of mRNAs and microRNAs is a novel mechanism of genetic exchange between cells. Nat Cell Biol. 2007; 9:654-9. [PubMed: 17486113]

32. Newsome TP, Asling B, Dickson BJ. Analysis of Drosophila photoreceptor axon guidance in eyespecific mosaics. Development. 2000; 127:851-60. [PubMed: 10648243]

33. Alberts, B.; Johnson, A.; Lewis, J.; Raff, M.; Roberts, K.; Walter, P. Molecular Biology of the Cell. 5. Garland Science; London: 2007.

34. Van Veldhoven PP, Baumgart E, Mannaerts GP. Iodixanol (Optiprep), an improved density gradient medium for the iso-osmotic isolation of rat liver peroxisomes. Anal Biochem. 1996; 237:17-23. [PubMed: 8660531]

35. Aleman LM, Doench J, Sharp PA. Comparison of siRNA-induced off-target RNA and protein effects. RNA. 2007; 13:385-95. [PubMed: 17237357]

36. Leung AK, Calabrese JM, Sharp PA. Quantitative analysis of Argonaute protein reveals microRNA-dependent localization to stress granules. Proc Natl Acad Sci U S A. 2006; 103:18125-30. [PubMed: 17116888] 

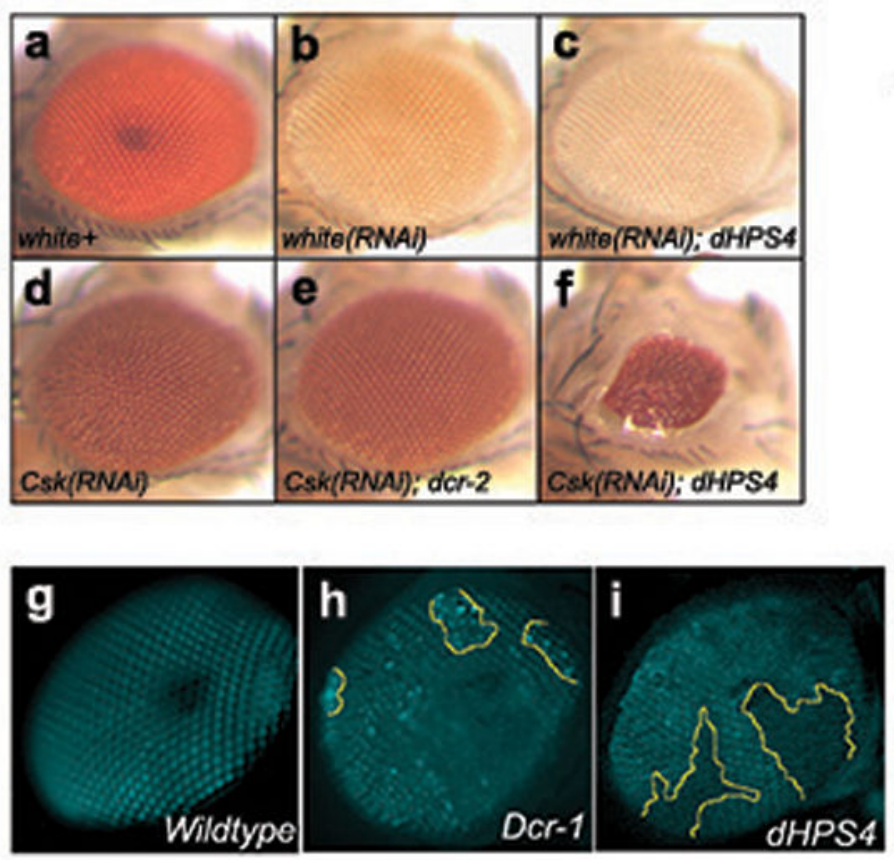

$\mathbf{k}$
$1 \mathrm{~kb}$
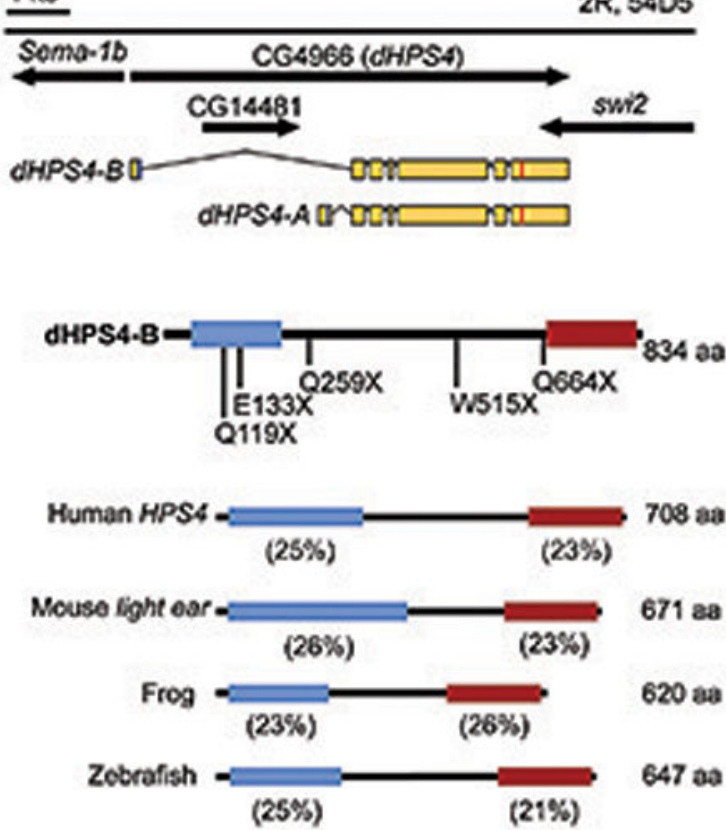

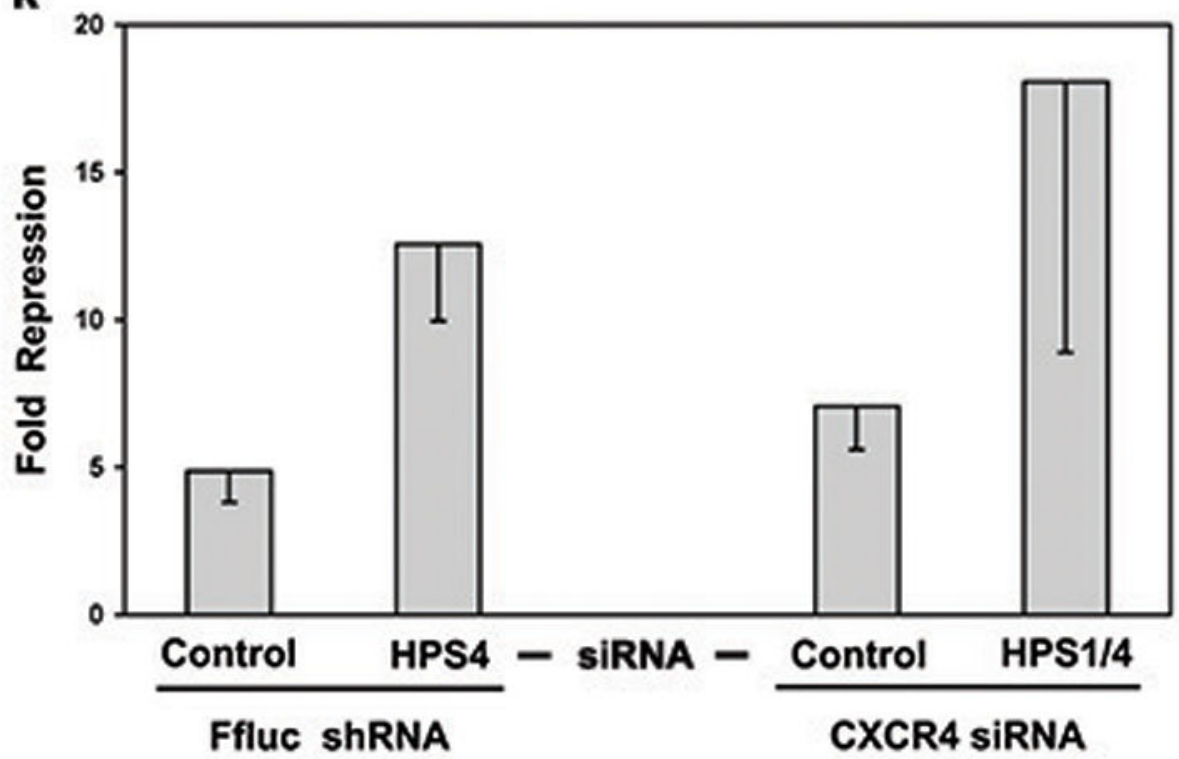

Figure 1. Loss of HPS4 enhances miRNA- and siRNA-mediated silencing a-c, Silencing of the white gene by GMR-wIR hairpin RNA is enhanced in a complementation group of mutations that maps to the $d H P S 4$ locus. Eyes from a wild-type adult (a), a white(RNAi) adult (b), a white(RNAi) adult in a mutant $d H P S 4$ background (c). Stronger silencing generates a white eye. d-f, Silencing of the Csk gene by Csk hairpin RNA is enhanced in $d H P S 4$ mutants. Eyes from a $C s k(\mathrm{RNAi})$ adult (d), a $C s k$ (RNAi) adult in a mutant dicer-2 (dcr-2) background (e), and a $C s k(\mathrm{RNAi})$ adult in a mutant $d H P S 4$ background (f). The mispatterning of the eye caused by $C s k$ (RNAi) was restored in a $d c r-2$ 
mutant lacking RNAi, and was strongly enhanced in the $d H P S 4$ mutant. g-i, Eyes from adults expressing GFP under control of the Brd $3^{\prime}$ UTR. (g) A non-mosaic wildtype eye. (h) Mosaic eye with presumed dicer-1 (dcr-1) mutant cells as outlined in yellow, and other cells are wildtype. Impaired miRNA processing in $d c r-1$ cells leads to de-repressed GFP expression. (i) Mosaic eye with presumed dHPS4 mutant cells as outlined in yellow, and other cells are wildtype. GFP expression is more strongly repressed. $\mathbf{j}$, The $d H P S 4$ gene and protein. Top, the $d H P S 4$ transcription unit. Positions of start and stop codons are indicated by vertical blue and red lines, respectively. Bottom, the protein products showing conserved domains. The SNARE-like longin domain is found within the conserved amino-terminal domain. The positions of the five mutations are indicated, all generating premature stop codons (X). Vertebrate orthologues are shown below, with conserved domains and percent amino acid identity to the Drosophila protein indicated. k, Depletion of HPS4 enhances siRNA-mediated silencing in HeLa cells. Silencing of a $P p$-luciferase reporter was triggered by an shRNA vector (left), and silencing of a Renilla-luciferase reporter gene was triggered by a CXCR4 siRNA (right). They were co-transfected with a control siRNA. Alternatively, they were co-transfected with either siRNA HPS4-882 (left) or siRNAs HPS1-242 and HPS4-1348 (right). Error bars represent one standard deviation. 

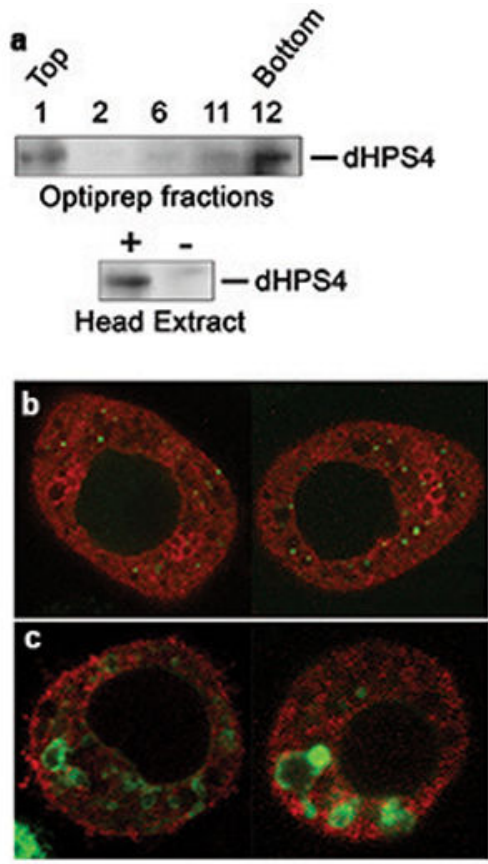

d
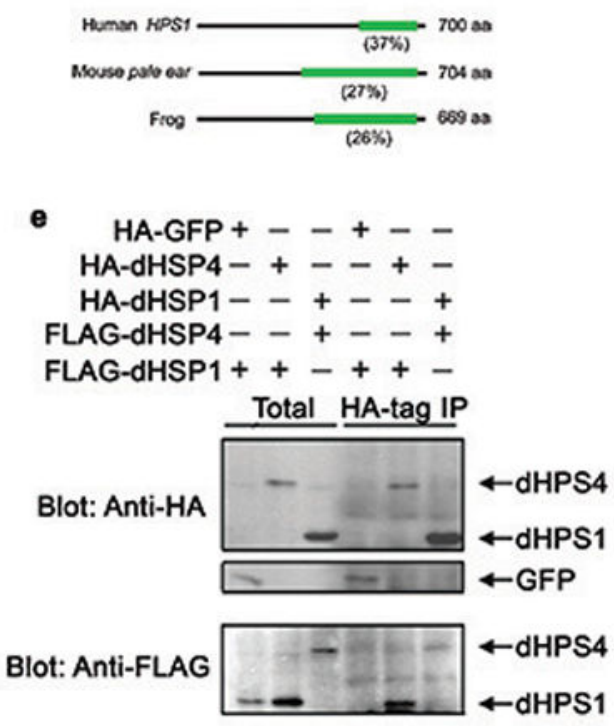

Figure 2. Characterization of dHPS4 and dHPS1

a, Top, Western blot of dHPS4 protein from wildtype adult head extract after fractionation by OptiPrep density gradient centrifugation. Selected fractions are shown. Fraction 1 at the top contains membrane-associated proteins, as indicated by the selective presence of dGM130 Golgi protein in Fraction 1 of parallel blots. Bottom, Western blot of dHPS4 protein from wildtype (+) and dHPS4 mutant (-) head extract. b. Two representative S2 cells visualized by confocal microscopy for HA-dHPS4 (red) and GFP-Me31b (green) proteins. GFP-Mei31b highlights GW-bodies. Confocal sections are high resolution (2048×2048 pixels from 100X objective) and ultra-thin $(0.4 \mu \mathrm{m})$. Parallel analysis of the same stable cell line without induction and naive $\mathrm{S} 2$ cells with induction confirmed that the 
anti-HA staining was dependent upon the presence of the MT>HA-dHPS4 gene and the metal inducer. These two cells represent a $n>50$ sample set. c, Two representative $\mathrm{S} 2$ cells visualized by confocal microscopy for HA-dHPS4 (red) and Lamp1-GFP (green) proteins. The dHPS4 protein surrounds Lamp1-positive endosomes and lysosomes. d, Domain structure of the HPS1 protein family. A conserved domain unique to the family is shown in green, and percent amino acid sequence identity between each orthologue and Drosophila HPS1 (CG12855) is shown in parentheses. Lengths of vertebrate orthologues are given in amino acids (aa). dHPS1 is 596 amino acids in length. e, Co-immunoprecipitation (Co-IP) of epitope-tagged dHPS4 and dHPS1 from transfected S2 cells. All IPs were performed on HA-tagged proteins, and FLAG-tagged proteins were probed for co-IP. HA-GFP was used as a control for IP specificity. 

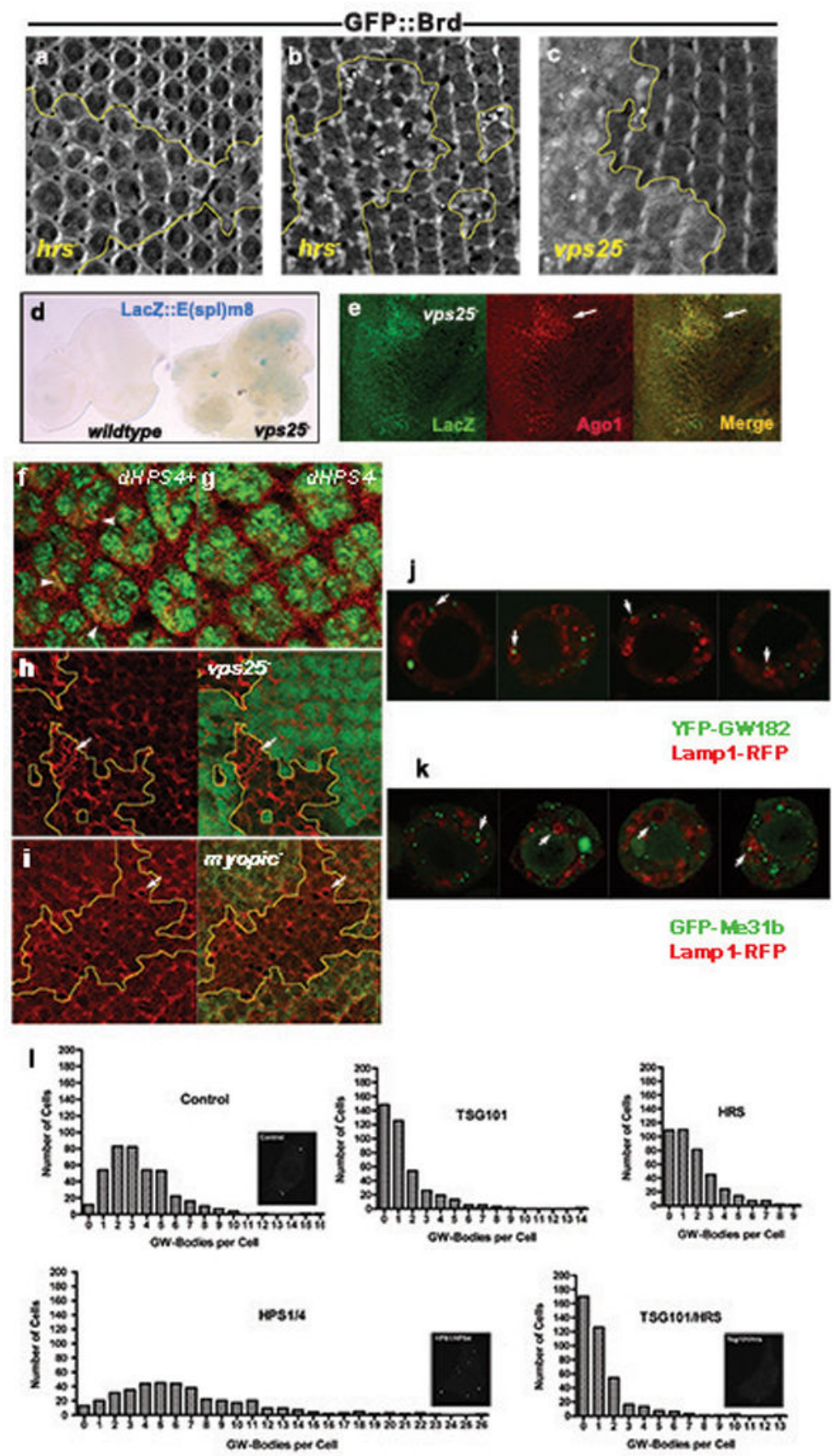

Figure 3. MVBs are sites of miRNA-mediated silencing a-c, Expression of the GFP::Brd reporter of miRNA silencing in pupal eyes that contain clones of $h r s^{D 28}(\mathbf{a}, \mathbf{b})$, and $v p s 25^{A 3}$ (c) mutant cells. Presumed clone boundaries are marked in yellow. Two hrs clones are shown at different $z$-planes to highlight GFP up-regulation in cone cells (a) and pigment cells (b). d-e, Expression of a lacZ::E(spl)m8 reporter of miRNA silencing in larval eye discs that contain $v p s 25^{A 3}$ clones. (d) Whole eye discs that were stained with X-Gal, with the left disc lacking any clones and the right disc containing $v p s 25^{A 3}$ clones. (e) A region of an eye disc with vps $25^{A 3}$ clones stained for the lacZ::E(spl)m8 reporter protein (green) and Ago1 protein (red). Note the correlation 
between enhanced lacZ::E(spl)m8 expression and Ago1 concentration in the presumed clones. $\mathbf{f}-\mathbf{i}$, Larval eye discs were stained for Ago1 protein (red) to detect miRISC distribution. Magnified views of wildtype (f) and $d H P S 4^{W 515 X}$ mutant (g) eye discs counterstained for nuclei (green). Perinuclear Ago1 localization in wildtype cells is highlighted with arrowheads. This localization is lacking in the mutant cells. Eye discs containing clones of $v p s 25^{A 3}(\mathbf{h})$, and myopic ${ }^{T 612}$ (i) mutant cells. In these genetically mosaic eyes, mutant cells are visualized by lack of expression of a marker gene (green). Yellow lines mark boundaries of clones. Mutant cells exhibit concentrated Ago1 around large vesicles (arrows). j-k, S2 cells showing Lamp1-RFP (red) that marks MVBs and lysosomes. Cells have been transfected with YFP-GW182 (j) or GFP-Me31b (k) to highlight GW-bodies (green). Note selected examples (arrows) of bodies juxtaposed with Lamp1positive membranes. I, Distributions of Ago2-GFP cytoplasmic bodies per cell in HeLa cell populations that underwent different siRNA treatments. Indicated in each plot are the siRNAs that were incubated with cells prior to counting GW-body numbers. Inset images are single HeLa cells from selected treatments showing Ago2-GFP bodies. 

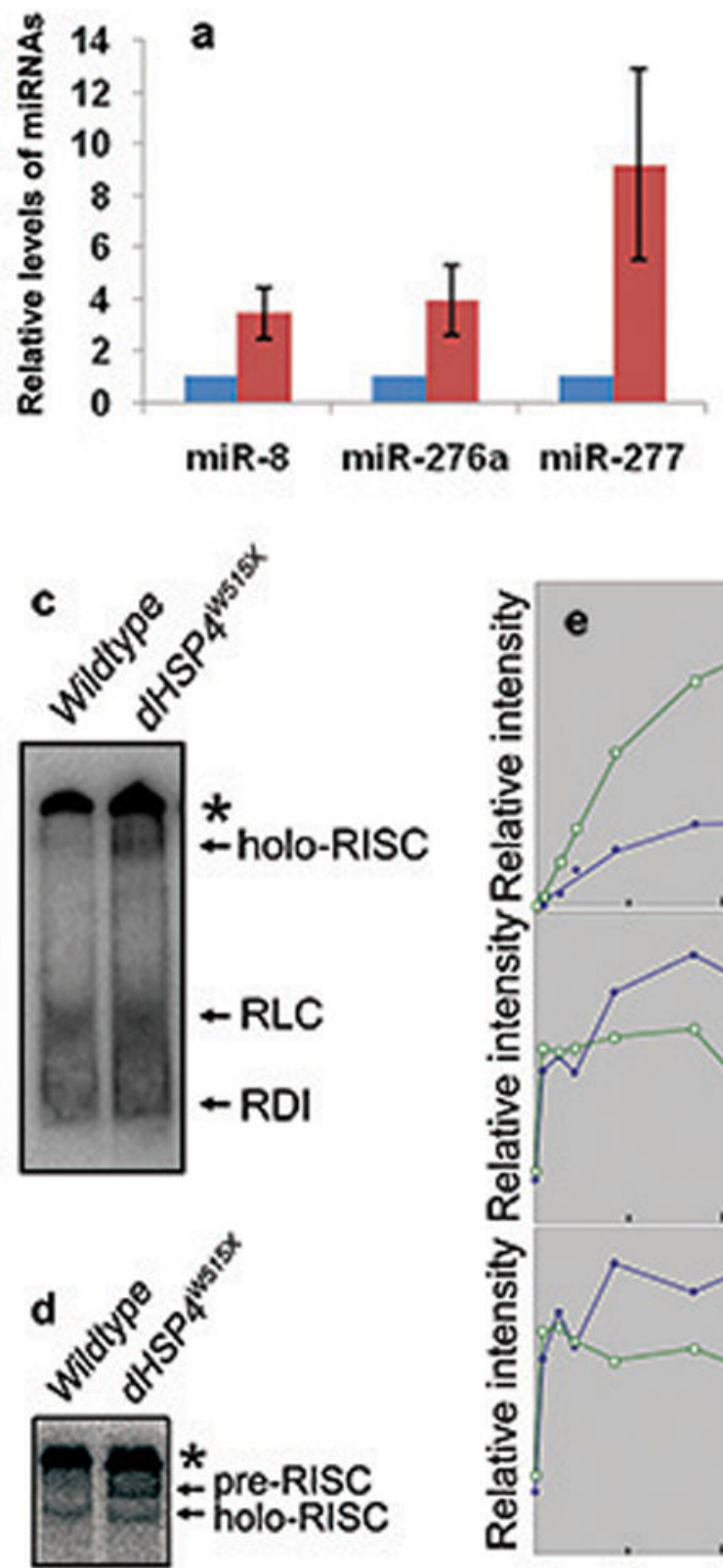

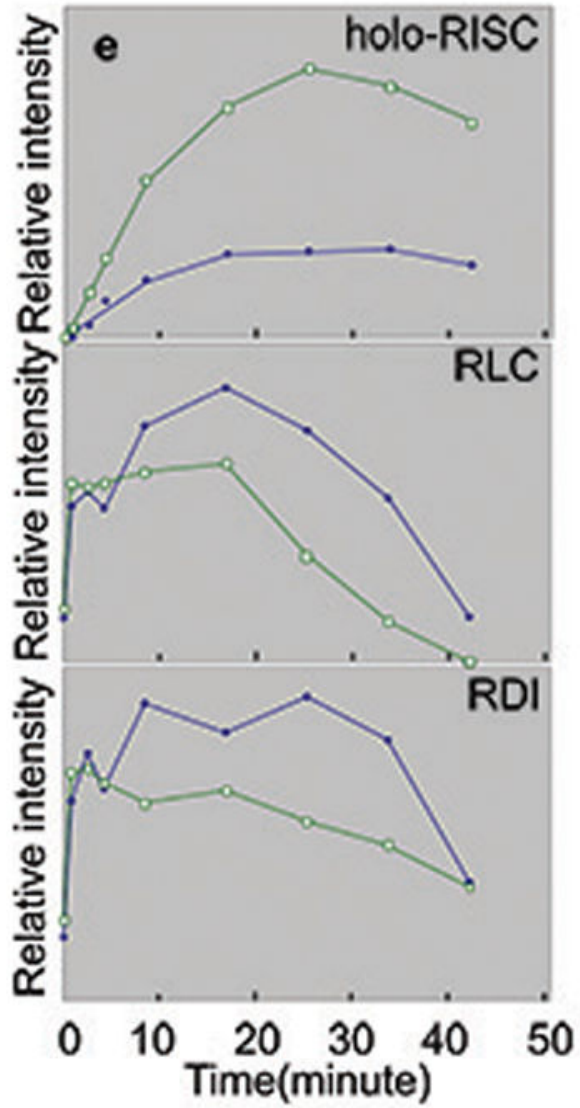

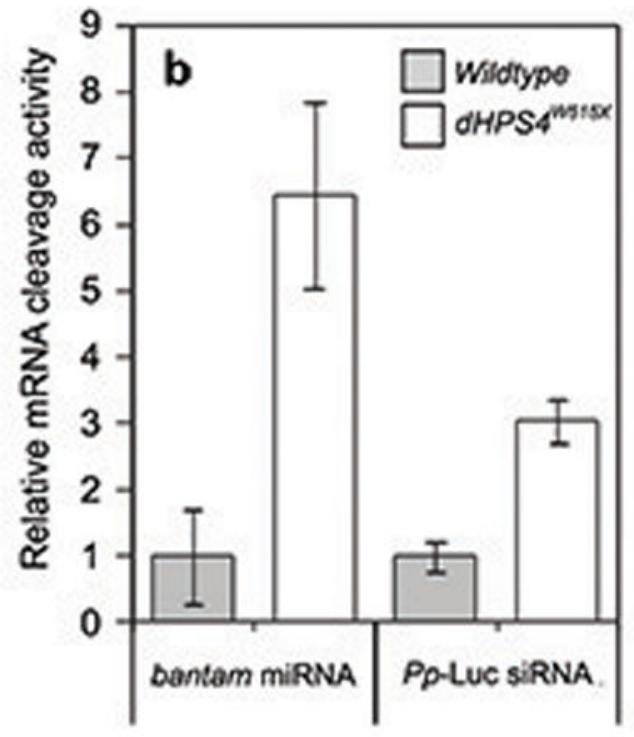

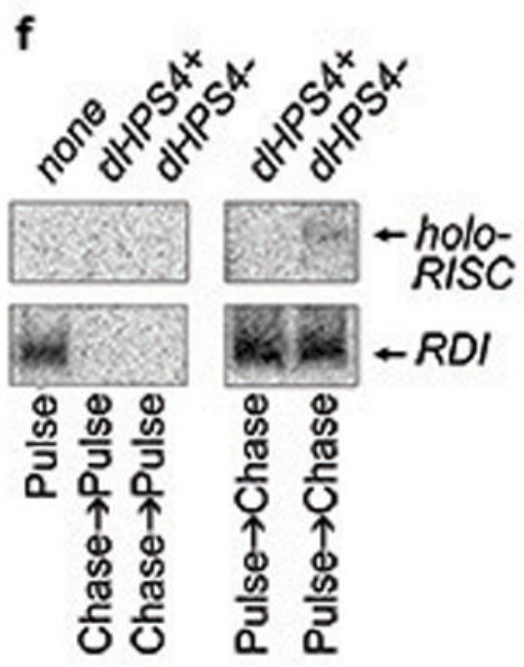

Figure 4. RISC loading of small RNAs is dependent upon MVBs

a, Quantitated levels of miR-8, miR-276a, and miR-277 miRNAs that coimmunoprecipitated with Ago1 protein from extracts of wildtype and $d H P S 4^{W 515 X}$ mutants. RNAs were detected by splinted ligation and were normalized to the amount of Ago1 immunoprecipitated in each sample. Data represent the mean \pm standard error of the normalized values from four independent experiments, relative to wildtype. b, Activity of RISC complexes in head extracts that were loaded with exogenous bantam miRNAs or $P p$ luc siRNAs. Cleavage of target mRNA with a sequence perfectly complementary to either 
the miRNA or siRNA was quantified. End-point cleavage activity of $d H P S 4$ mutant extracts is expressed relative to wildtype extracts. Error bars show standard deviations. c, Loading of holo-RISC in wildtype and $d H P S 4^{W 515 X}$ mutant head extracts after addition of labelled $P p$ luc siRNA. RDI, RLC and holo-RISC complexes are indicated in this native gel. An asterisk marks material too large to enter the gel. d, Loading of pre-RISC in $d H P S 4^{W 515 X}$ mutant head extract after addition of a labelled siRNA that contains a single base mismatch. This siRNA enables detection of pre-RISC, which is otherwise not observed in the assay. e, Time course following RDI, RLC, and holo-RISC loading with wildtype (blue diamonds) and dHPS4 ${ }^{W 515 X}$ mutant (green squares) extracts. f, Pulse (purified RDI added with labelled siRNA) and Chase (head extract added with excess unlabelled siRNA) mixtures were added to reactions in order as indicated. Native gel analysis visualized RDI and holo-RISC complexes associated with labelled siRNA. Genotypes of extracts are indicated above. 


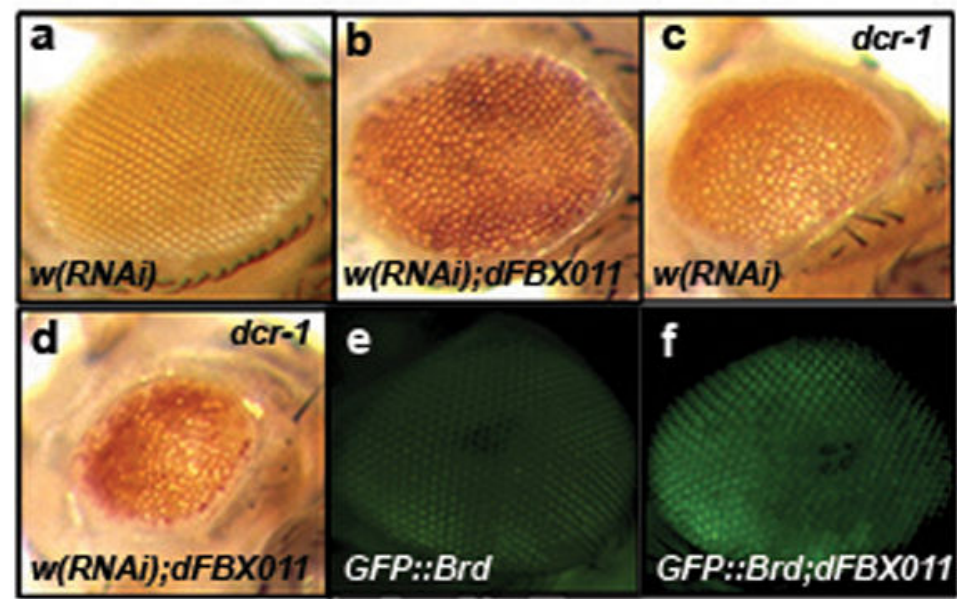

g

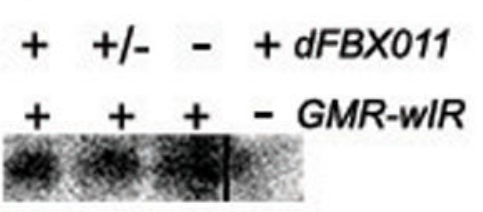

h $\quad$ IFBX011

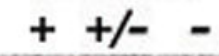

miR-2abc
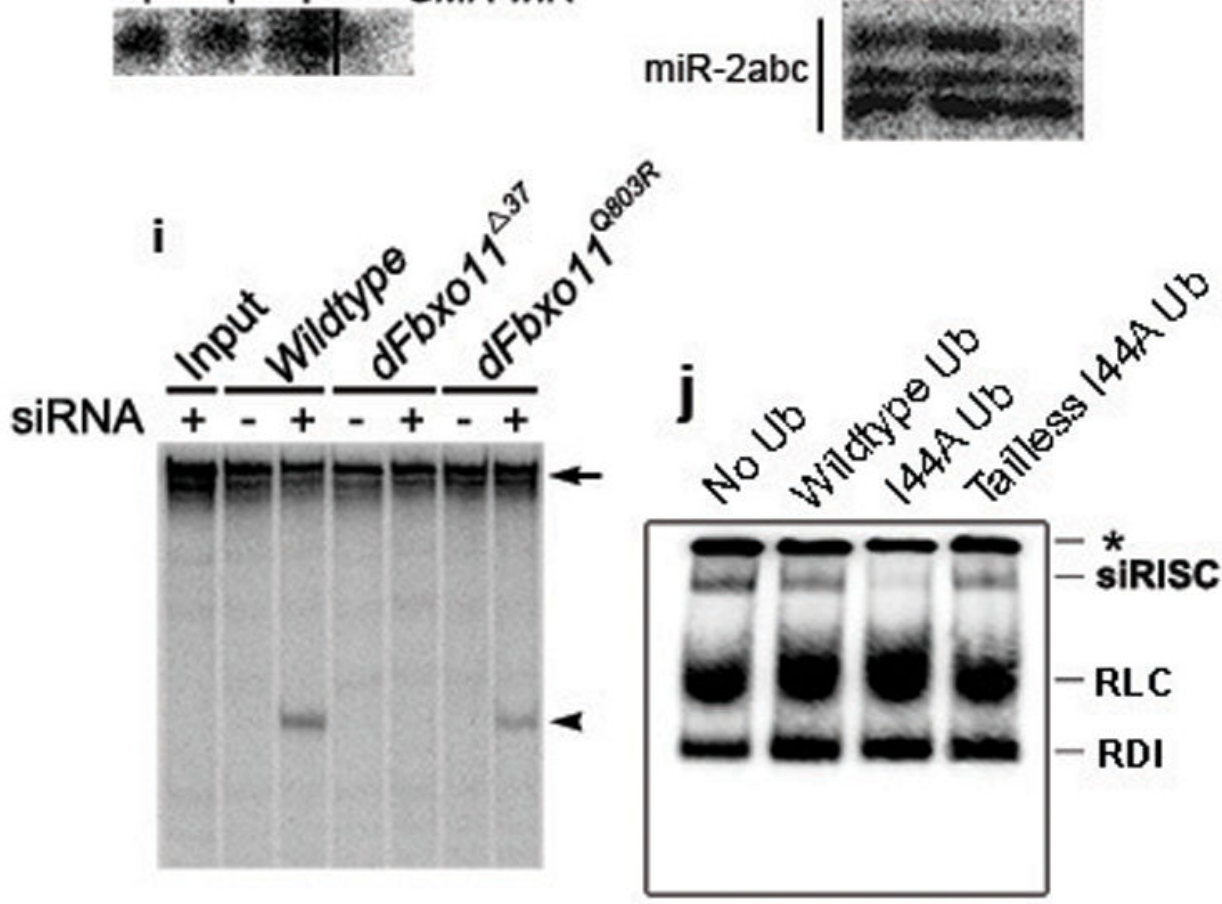

Figure 5. Protein ubiquitination is required for RNA silencing and RISC loading a-b, Eyes of white ${ }^{+}$adult flies with one copy of GMR-wIR. (a) The white gene is partially silenced, resulting in an orange eye colour in a $d F B X 011^{+}$background. (b) A fly homozygous for $d F B X 011^{Q 803 R}$ showing a redder eye colour that indicates impaired siRNAmediated silencing. c-d, Eyes of adults with one copy of GMR-wIR and mutant for $d c r-1$. (c) Loss of $d c r-1$ results in a smaller eye and partially impaired silencing of white. (d) An eye homozygous for $d c r-1 d F B X 011^{Q 803 R}$ shows greater impairment of white silencing and enhanced eye reduction due to genetic interaction with $d c r-1$. e-f, Eyes of adults carrying the GFP ::Brd reporter for miRNA-mediated silencing. (e) An adult showing normal GFP 
expression when silenced. (f) An adult with clones of $d F B X 011^{Q 803 R}$ mutant cells in its eye. GFP silencing is inhibited in $d F B X 011^{Q 803 R}$ mutant clones, resulting in strongly variegated GFP expression. g, Northern blot for white siRNAs from adults with indicated genotypes. The $d F B X 011$ mutant allele used was $d F B X 011^{Q 803 R}$. $\mathbf{h}$, Northern blot for miR-2 RNAs from adult heads with indicated $d F B X 011$ genotypes. There are three isoforms of miR-2, which can be seen on the blot. i, siRISC activity in wildtype and $d F B X 011$ mutant extracts that were loaded with exogenously added $P p$-luc siRNA. The unreacted $P p$-luc mRNA substrate and the $5^{\prime}$ cleavage product are indicated by an arrow and arrowhead, respectively. j, A ubiquitin mutant (I44A) protein with defects in protein-protein interactions exerts a dominant-negative effect on loading of siRISC with labelled $P p$-luc siRNA. The "tailless" negative control carries a four-amino-acid C-terminal truncation and is unable to enter the ubiquitin conjugation pathway. 